\title{
A Review of Electrowetting on Dielectric Applications of Carbon Nanotubes
}

Manasi Raje*

Shield Diagnostics, Santa Clara, California, USA

*Corresponding author: Manasi Raje, Microfluidics R\&D Engineer, Shield Diagnostics, Santa Clara, California, USA, Email: mraje@uci.edu

\section{Mini Review}

Volume 3 Issue 4

Received Date: September 19, 2018

Published Date: October 04, 2018

DOI: $10.23880 /$ nnoa- 16000151

\section{Abstract}

The unique properties carbon nanotubes (CNTs) have shown potential for use in electrowetting on dielectric (EWOD) technology. The conductivity, thermal and optical properties of CNTs can be exploited to cater to various needs in the many layers of a EWOD device. CNTs have been used as conductive layers, thermal management elements, transparent conductive layers, chemical and biological sensors. This mini review explores the various attempts of exploiting the excellent electrical and physical properties CNTs in EWOD and similar applications. The review thus identifies the overlap in these two fields that indicates a strong future opportunity to use CNTs in EWOD systems.

Keywords: Carbon Nanotubes (CNTs); Electrowetting on Dielectric (EWOD); Microscale; Hydrophobic Material

\section{Introduction}

\section{Electrowetting on Dielectric Devices}

Electrowetting on dielectric (EWOD) is a popular technology used in digital microfluidic devices to move microscale quantity of liquid on a substrate with electrodes [1]. A typical EWOD device consists of a substrate made of glass, silicon, paper, polyimide and more possible choices of material. The substrate is patterned with several electrodes and is coated with a thin uniform layer of a dielectric material. The entire device is then coated with a hydrophobic material to obtain a high contact angle for liquid droplets as well as to avoid adsorption of biomolecules. On application of high voltage to the electrodes an electric field is established over the corresponding electrode which reduces the contact angle of the liquid with the hydrophobic surface. The reduction in contact angle causes the liquid to "wet" the electrode and thus sticking to the surface for the period during which electric field is prevalent. The change in contact angle is given by the Young-Lippman equation (Equation 1) [2].

$$
\cos \theta_{v}=\cos \theta_{0}+\frac{1}{\gamma_{L G}} \cdot \frac{\varepsilon_{r} \varepsilon_{0}}{2 t} V^{2}
$$

Upon removal of the high voltage and therefore electric field the droplet "de-wets" the surface. This wetting and dewetting behavior can be used to move droplet form one electrode to the other thereby achieving transport of liquid on the microfluidic chip. Figure 1 shows the working of a parallel plate EWOD device. EWOD microfluidic technology has been used in many fields such as biofuels [3], blood-based assays [4] and many more. A growing demand of this technology is observed in applications involving flexible displays, electronic skin, etc $[5,6]$. Such applications require the 


\section{Nanomedicine \& Nanotechnology Open Access}

device to be transparent and have low thickness of the various layers on the chip. Further, an interest in using novel materials for the conducting electrodes and dielectrics is also observed due to the high demand in conductivity and physical properties.

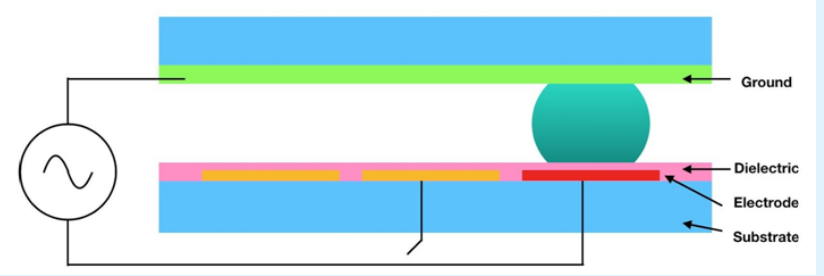

Figure 1: Cross-sectional view of a typical two plate EWOD device showing all the layers.

\section{Carbon Nanotubes Properties and Fabrication}

Carbon nanotubes (CNT) is an emerging and promising technology. Carbon nanotubes are very tiny tube like structures made of either single of multi walled graphite. The properties of CNTs depend on their structure. A layer of graphite is rolled up to obtain a nanotube and the properties that the nanotube will exhibit depend on the direction of rolling up. These nanostructures have excellent electrical properties and various additional physical properties that have proved to be beneficial for use in EWOD devices and other applications as well. Theory and experiments show that CNTs have high conductive and high mobility [7]. The attractive physical properties of nanotubes such as thermal conductivity [8], malleability, and transmittance [7] make it a good choice for use in a variety of applications. A 2D network, often referred to as a thin film, made of randomly distributed CNTs can be regarded as a novel material [7]. The thin film obtained from CNTs can be essentially used for many applications and nanelectrodes is one of those [7]. Surface electrowetting properties of carbon nanotubes for the inside of nanotubes as well as for outside of the nanotubes have been studied $[9,10]$. These studies indicate that CNTs have potential in nanofluidics, drug delivery and nanoscale energy conversion [11].

An understanding of the level of complexity of fabrication can be useful in predicting the potential future directions for the use of CNTs in EWOD devices. Thin film CNTs can be obtained by either direct growth of CNTs on the substrate or by solution based deposition [7]. In the case of direct growth the CNTs are produced by three major methods: chemical vapor deposition (CVD), arcdischarge and laser ablation [7]. For solution based methods, nanotubes are first suspended in a dispersion aid which can be a detergent, polymer, organic solvent, or other application specific dispersion aids [7]. The solution based methods are preferred when low temperature is crucial considering the substrate properties whereas the direct growth methods are suitable for achieving high conductivity and film purity [7]. Park, et al. demonstrated a novel method of deposition of SWCNT films using microfluidic laminar flow. By parallel flowing two streams of methanol and surfactant suspended CNTs, they exploited the affinity of methanol as a solvent to the surfactant that us used to suspend CNTs thereby forming a flat layer of CNTs as a result of diffusion between two streams in laminar flow [12].

\section{Utility of CNTs in EWOD}

A number of groups have utilized the transparency of ITO as a conductive material, gold as a stretchable material. However, some attempts at using graphene or carbon nanotubes have shown promise to exploit the electrical as well as physical properties for making EWOD devices [5,6]. Xuebin Tan, et al. studied the potential of thin layered graphene as the electrode layer in EWOD devices. They established that graphene is a strong candidate to be used in electrowetting on dielectric devices compared to other common conductive materials such as gold, for properties like higher breakdown voltage, electrical conductivity optical transparency and flexibility, as well as lower cost [6]. Gruner, et al. explored the use of SWCNTs as the conductive electrode layer in a EWOD microfluidic device. The group fabricated EWOD devices with a spray coated CNT films as the conductive layer, contact angle and droplet translation was tested to evaluate the performance of their nanotube EWOD devices. They demonstrated carbon nanotube electrodes in EWOD devices having indistinguishable performance when compared to ITO based electrode EWOD devices. Chen et al. grew CNT structures on the ground plate in their EWOD device in order to exploit the thermal conductivity of CNTs for the purpose of thermal management on microfluidic chip [8]. Lipomi, et al. also used the technique of spray coating for obtaining a conductive layer of CNTs [13] to make stretchable wearable pressure sensors indicating the strong potential of CNTs for easy fabrication of wearable electronics. Facilitating one more step towards development of low cost microfluidic devices, Ko, et al. have thoroughly discussed the opportunity of printing CNT electrodes on paper for digital microfluidic application using an inkjet 


\section{Nanomedicine \& Nanotechnology Open Access}

printer [14]. CNTs properties are also used as a research tool to study the performance of electrowetting in EWOD systems. For example, Wang et al. used the ability to make nanostructures using CNTs on for parylene dielectric layer in order to compare the effect of roughness in contact angle saturation [15]. Shahini, et al. used the CNTs as electroporation electrodes for cell lysis [16]. Previously Shih, et al. demonstrated a versatile EWOD device for synthetic biology that included a region on the EWOD chip with bare gold-chromium electrodes for electroporation of cells [3]. Further, more complex applications of CNTs include detection of biomolecules like enzymes, proteins and DNA. The incorporation of carbon nanotubes has the potential to address a variety of long-standing issues with respect to bio-sensing [17]. Similarly, EWOD technology has been attractive due to its promising potential in biological, biochemical and biomedical applications [18].

\section{Discussion}

A number of reports that used the properties of CNTs for different applications have been reviewed and the overlap of functionality and application has been identified in this short review.

The tremendous progress in the fabrication techniques and research of CNTs has proved the strong potential for utility in electrowetting based nanoscale and microscale devices. EWOD is a technology that is used in many fields including microelectronics, bio applications, chemical sensing and many more. The incorporation of CNTs to EWOD has been successful and rewarding and holds promise to spread the reach of EWOD in even more areas of science and technology.

\section{References}

1. Nelson WC, Chang-Jin CJK (2012) Droplet Actuation by Electrowetting-on-Dielectric (EWOD): A Review. Journal of Adhesion Science and Technology 26(1217): 1747-1771.

2. Lin YY, Evans RD, Welch E, Hsu BN, Madison AC, et al. (2010) Low Voltage Electrowetting-on-Dielectric Platform using Multi- Layer Insulators. Sens Actuators B Chem 150(1): 465-470.

3. Shih SCC, Goyal G, Kim PW, Koutsoubelis N, Keasling JD, et al. (2015) A Versatile Microfluidic Device for Automating Synthetic Biology. ACS Synthetic Biology 4(10): 1151-1164.
4. Mousa NA, Jebrail MJ, Yang $H$, Abdelgawad $M$, Metalnikov P, et al. (2009) Droplet-Scale Estrogen Assays in Breast Tissue, Blood, and Serum. Science Translational Medicine 1(1): 1-2.

5. Liangbing $\mathrm{Hu}$, Gruner G, Gong J, Chang-Jin CJK, Hornbostel B (2007) Electrowetting devices with transparent single-walled carbon nanotube electrodes. A Applied Physics Letters 90(9): 093124.

6. Tan X, Zhou Z, Cheng MMC (2012) Electrowetting on dielectric experiments using graphene. Nanotechnology 23(37).

7. Hu L, Hecht DS, Grüner G (2010) Carbon Nanotube Thin Films: Fabrication, Properties, and Applications. Chem Rev 110(10):5790-5844.

8. Cheng JT, Chen CL (2010) Adaptive Chip Cooling Using Electrowetting on Coplanar Control Electrodes. Nanoscale and Microscale Thermophysical Engineering 14(2): 63-74.

9. Chen JY, Kutana A, Collier CP, Giapis KP (2005) Electrowetting in Carbon Nanotubes. Science 310(5753): 1480-1483.

10. Liu Z, Zheng K, Hu L, Liu J, Qiu C, et al. (2010) SurfaceEnergy Generator of Single-Walled Carbon Nanotubes and Usage in a Self-Powered System. Adv Mater 22(9): 999-1003.

11. Zhao YP, Wang Y (2013) Fundamentals and Applications of Electrowetting: A Critical Review. Rev Adhesion Adhesives 1(1): 114-174.

12. Park JU, Meitl MA, Hur SH, Usrey ML, Strano MS, et al. (2006) In Situ Deposition and Patterning of SingleWalled Carbon Nanotubes by Laminar Flow and Controlled Flocculation in Microfluidic Channels. Angew Chem Int Ed 45(4): 581-585.

13. Lipomi DJ, Vosgueritchian M, Tee BCK, Hellstrom SL, Lee JA, et al. (2011) Skin-like pressure and strain sensors based on transparent elastic films of carbon nanotubes. Nature Nanotechnology 6(12): 788-792.

14. Ko H, Lee J, Kim Y, Lee B, Jung CH, et al. (2014) Active Digital Microfl uidic Paper Chips with Inkjet-Printed Patterned Electrodes. Adv Mater 26(15): 2335-2340.

15. Wang Z, Ou Y, Lu TM, Koratkar N (2007) Wetting and Electrowetting Properties of Carbon Nanotube 


\section{Nanomedicine \& Nanotechnology Open Access}

Templated Parylene Films. The Journal of Physical Chemistry B 111(17): 4296-4299.

16. Shahini M, Yeow JTW (2013) Cell electroporation by CNT-featured microfluidic chip. Lab Chip 13(13): 2585-2590.

17. Kim SN, Rusling JF, Papadimitrakopoulos F (2007) Carbon Nanotubes for Electronic and Electrochemical
Detection of Biomolecules. Adv Mater 19(20): 32143228.

18. Lin TH, Yao DJ (2012) Applications of EWOD Systems for DNA Reaction and Analysis. Journal of Adhesion Science and Technology 26(12-17):1789-1804.

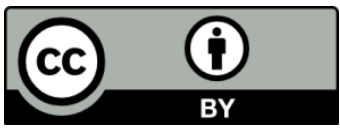

